\title{
NUCLEOSYNTHESIS IN CLASSICAL NOVAE
}

\author{
MARGARITA HERNANZ \\ Institut d'Estudis Espacials de Catalunya, IEEC, and Instituto de Ciencias del \\ Espacio (CSIC), Edifici Nexus, C/Gran Capità, 2-4, E-08034 Barcelona, Spain \\ JORDI JOSÉ \\ Institut d'Estudis Espacials de Catalunya, IEEC, and Departament de Física $i$ \\ Enginyeria Nuclear (UPC), Avda. Víctor Balaguer, s/n, E-08800 Vilanova i la \\ Geltrú (Barcelona), Spain \\ ALAIN COC \\ Centre de Spectrométrie Nucléaire et de Spectrométrie de Masse, IN2P3-CNRS, \\ Université de Paris Sud, Bâtiment 104, F-91405 Orsay Cedex, France
}

\begin{abstract}
A general review of the relevance of classical novae for the chemical evolution of the Galaxy, as well as for Galactic radioactivity is presented. A special emphasis is put on the pioneering work done by Jim Truran in this field of research. The impact of recent developments in nuclear astrophysics on nova nucleosynthesis, together with the prospects for observability of novae radioactivities with the INTEGRAL satellite are discussed.
\end{abstract}

\section{Introduction}

Classical novae are explosions occurring on the top of white dwarfs accreting hydrogen-rich matter in close binary systems, of the cataclysmic variable type. The transfer of matter results from Roche lobe overflow from the main sequence companion star. The accreted hydrogen is compressed up to degenerate conditions, leading to a thermonuclear runaway. Explosive hydrogen burning occurs via the $\mathrm{CNO}$ cycle, out of equilibrium because radioactive nuclei with timescales longer than the evolutionary timescale are produced. These nuclei are transported by convection to the outer envelope, where they are preserved from destruction until they decay. This late decay is the final responsible of the envelope expansion and increase in luminosity characteristic of nova outbursts.

It is important to stress that Jim Truran, with Sumner Starrfield and collaborators, was a pioneer in the study of thermonuclear explosions. Starrfield, Truran, Sparks and Kutter wrote a series of papers, starting in the early 7f's which presented the first hydrodynamic computations of classical novae 1 . phase was not followed. One of the main conclusions was that the envelope

hernanz: submitted to World Scientific on November 19, 2018 
should be enhanced in CNO nuclei in order to power the nova outburst. This is still a valid fact, and it poses severe problems to the current theory, because there isn't a clear understanding of the mixing mechanism responsible for that enhancement. In 1978, another group also developed a hydro code to study nova explosions $\mathbf{6}$, with similar limitations concerning the accretion phase. It is also worth mentioning that Jean Audouze also contributed with Jim Truran to the progress in the nova field, since he studied the properties of the hot CNO burning 6 , which (although not "so hot") is crucial for the explosion.

Classical nova explosions are very common: $\sim 35$ per year in our Galaxy, from which only 3 to 5 are discovered. They release large amounts of energy, $\sim 10^{45} \mathrm{erg}$, but not large enough to have an impact on the dynamics of the interstellar medium, like supernovae. The small mass ejected (in the range $10^{-5}-10^{-4} \mathrm{M}_{\odot}$ ) implies that novae scarcely contribute to the chemical evolution of the Galaxy. If we adopt an ejected mass of $2 \times 10^{-5} \mathrm{M}_{\odot}$, a Galactic nova rate of $35 \mathrm{yr}^{-1}$ and an age of the Galaxy of $10^{10} \mathrm{yr}$, novae can account only for $\sim 1 / 3000$ of the gas and dust in the Galactic disk; this number is somehow a lower limit, since the ejected mass deduced from observations of some novae is larger than the typical theoretical value adopted here. However, novae are the main producers of some particular isotopes $\left({ }^{13} \mathrm{C},{ }^{15} \mathrm{~N}\right.$ and $\left.{ }^{17} \mathrm{O}\right)$, they can help to explain the ${ }^{7} \mathrm{Li}$ versus metallicity relation in the Galaxy and they contribute to the radioactivity of the Galaxy, through the ejection of medium and long-lived radioactive isotopes (such as ${ }^{7} \mathrm{Be},{ }^{22} \mathrm{Na}$ and ${ }^{26} \mathrm{Al}$ ). In addition, novae often form dust, which manifests through their IR emission; therefore they could explain the anomalous isotopic signatures measured in some presolar grains 6 . 20 (see also the excellent recent review 11 ).

\section{Novae and the chemical evolution of the Galaxy}

Novae can be classified in two types, according to their observed abundances. The often called "standard" novae are objects where enrichments in CNO nuclei have been reported, whereas the so-called "neon" novae show also enrichments in neon This class of novae was first studied hydrodynamically by Starrfield et al. 12, including the follow-up of the accretion phase. The two nova types are interpreted as the result of explosions on accreting carbonoxygen $(\mathrm{CO})$ and oxygen-neon $(\mathrm{ONe})$ white dwarfs 丹, respectively, because the chemical composition of the ejecta reflects that of the underlying white dwarf.

${ }^{a}$ Until recently, massive white dwarfs were called ONeMg white dwarfs, because they were thought to be made of these elements. But now it is known that these stars are almost devoid of magnesium and, consequently, should be called ONe white dwarfs

hernanz: submitted to World Scientific on November 19, 2018 
The reason is that some mixing between the accreted envelope (presumed to have close to solar composition) and the underlying core is required, in order both to power the explosion and to explain the observed enrichments in CNO and/or Ne. The question of the frequency of occurrence of QNe white dwarfs in classical novae was addressed by Truran and Livio 13, 44, who concluded that these white dwarfs can account for $\sim 1 / 3$ of all observed outbursts.

The mechanism responsible of the mixing has not been determined yet (shear mixing diffusion, convection). Recent two and three-dimensional calculations 15.16.17 address the convective mixing during the hydrodynamic phase following degenerate ignition of hydrogen. There isn't yet a clear way to mix and probably the mixing, or some part of it, should proceed in the previous hydrostatic accretion phase Diffusion during a number of succesive flashes has been also simulated 18.19. In this case, moderate enhancement can be obtained for some combinations of initial conditions (mass, luminosity, accretion rate); the remaining problem of this scenario is the handling of mass-loss during succesive flashes.

Until some clear mechanism is identified and understood, we 20 adopt a parametrization of the mixing with the underlying core (ranging from 25 to 75 $\%$, in order to explain the range of observed metallicities in novae ejecta; other authors 21.22 adopt only 50\%). In table 1 we show the main nucleosynthetic products of a handful of nova models, representative of both classes of novae (see José and Hernanz 20 for a larger sample). CO white dwarfs have masses up to $\sim 1.15 \mathrm{M}_{\odot}$, whereas $\mathrm{ONe}$ have larger masses (the exact limit between both types of degenerate cores, related to the previous evolution during the AGB phase, is still an open issue). The accretion rate adopted is $2 \times 10^{-10}$ $\mathrm{M}_{\odot} \cdot \mathrm{yr}^{-1}$ and the initial luminosity $10^{-2} \mathrm{~L} \odot$ (initial mixing $50 \%$ ). We show the ejected masses of the most overproduced isotopes $\left({ }^{13} \mathrm{C},{ }^{15} \mathrm{~N}\right.$ and $\left.{ }^{17} \mathrm{O}\right)$, together with ejected masses of medium and long-lived radioactive nuclei. In figure 1 we show the overproduction factors, with respect to solar, of all the isotopes contained in the ejecta of a $\mathrm{CO}$ and an ONe novae, of the same mass, in order to illustrate the impact of the chemical composition of the white dwarf. ${ }^{13} \mathrm{C},{ }^{15} \mathrm{~N}$ and ${ }^{17} \mathrm{O}$ are overproduced in both nova types by similar factors. ONe novae also eject Ne, required to fit the observations. The radioactive isotopes ${ }^{22} \mathrm{Na}$ and ${ }^{26} \mathrm{Al}$ are almost only produced in ONe novae, because of the presence of seed nuclei in the accreted envelope mixed with the core; this presence is necessary to power the $\mathrm{NeNa}-\mathrm{MgAl}$ reaction rate cycles responsible of the synthesis of ${ }^{22} \mathrm{Na}$ and ${ }^{26} \mathrm{Al}$ synthesis. On the other hand, ${ }^{7} \mathrm{Li}$ (coming from the radioactive ${ }^{7} \mathrm{Be}$ ) is produced in larger amounts in $\mathrm{CO}$ novae, because of the shorter duration of the accretion phase (with $\mathrm{T}$ smaller than $\sim 10^{8} \mathrm{~K}$ ), which is the critical one for ${ }^{3} \mathrm{He}$ survival, with ${ }^{3} \mathrm{He}$ being

hernanz: submitted to World Scientific on November 19, 2018 
essential for ${ }^{7}$ Be synthesis (see Hernanz et al. 23 for details). The production of ${ }^{7} \mathrm{Li}$ in novae was already predicted by the computations of Starrfield et al. 24 with their hydro code more than 20 years ago, but at the epoch they didn't follow the accretion phase, since they had the accreted envelope in place; therefore, a crucial phase for ${ }^{7} \mathrm{Li}$ synthesis was missed. The relevance of novae for the Galactic content of ${ }^{7} \mathrm{Li}$ is still an open issue. A rough estimate of their average contribution gives a maximum of the $20 \%$, but the important fact is at which stage of the Galactic evolution they contribute. In a recent paper 25 , it has been shown that ${ }^{7} \mathrm{Li}$ contribution from novae is required in order to reproduce the shape of ${ }^{7} \mathrm{Li}$ versus metallicity relationship, which grows at low metallicities.

Comparison between theoretical abundances in the ejecta and observed abundances in particular novae gives a quite good agreement 20 both for CO and $\mathrm{ONe}$ novae22, as well as a global comparison of average properties in $\mathrm{CO}$ novae 19.

\section{Novae and Galactic radioactivity}

The content of $\mathrm{CO}$ and $\mathrm{ONe}$ novae ejecta in radioactive isotopes is shown in table 1 for some significative models. As mentioned before, $\mathrm{CO}$ novae are more successful in producing ${ }^{7} \mathrm{Be}$, whereas ONe novae produce larger quantities of ${ }^{22} \mathrm{Na}$ and ${ }^{26} \mathrm{Al}$ than $\mathrm{CO}$ novae. This fact has important consequences for the $\gamma$-ray signaturesexpected for both types of novae, as shown in detail in our recent papers 2627: individual $\mathrm{CO}$ novae will preferentially emit photons of $478 \mathrm{keV}$ (with $\tau=77$ days), whereas ONe will emit $1275 \mathrm{keV}$ photons $(\tau=3.75$ yr). Concerning the cumulative emission, ONe novae can contribute to the Galactic content of ${ }^{26} \mathrm{Al}\left(\tau=10^{6} \mathrm{yr}\right)$, although their contribution is not the most important one: according to the observations of the Galactic $1809 \mathrm{keV}$ emission, made with the CGRO/COMPTEL instrument, this emission seems to be preferentially associated with a massive star population.

In the recent years, we have studied in detail the impact that uncertainties in nuclear reactions have on nova yields, specially for the radioactive ones 28.29, in order to make predictions of detectability of classical novae by the future ESA satellite INTEGRAL, to be launched in April 2002. Novae (ONe) up to distances of $\sim 2 \mathrm{kpc}$ could be detected through the ${ }^{22} \mathrm{Na}$ line at 1275 $\mathrm{keV}$, whereas $\mathrm{CO}$ novae should be at shorter distances to be detected through the ${ }^{7} \mathrm{Be}$ line at $478 \mathrm{keV} 26$. The most powerful emission of $\gamma$-rays from novae is the line emission at $511 \mathrm{keV}$ and the continuum at energies between 20 and $511 \mathrm{keV}$, related to $\mathrm{e}^{-}-\mathrm{e}^{+}$annihilation, with the positrons coming from the very short-lived isotopes ${ }^{13} \mathrm{~N}$ and ${ }^{18} \mathrm{~F}$. This emission could be detected up to 3

hernanz: submitted to World Scientific on November 19, 2018 
Table 1. Nucleosynthesis in CO and ONe novae

\begin{tabular}{|c|c|c|c|c|c|c|c|}
\hline Nova & $\mathrm{M}_{\mathrm{wd}}\left(\mathrm{M}_{\odot}\right)$ & ${ }^{13} \mathrm{C}\left(\mathrm{M}_{\odot}\right)$ & ${ }^{15} \mathrm{~N}\left(\mathrm{M}_{\odot}\right)$ & ${ }^{17} \mathrm{O}\left(\mathrm{M}_{\odot}\right)$ & ${ }^{7} \mathrm{Be}\left(\mathrm{M}_{\odot}\right)$ & ${ }^{22} \mathrm{Na}\left(\mathrm{M}_{\odot}\right)$ & ${ }^{26} \mathrm{Al}\left(\mathrm{M}_{\odot}\right)$ \\
\hline $\mathrm{CO}$ & 0.8 & $7.7 \times 10^{-6}$ & $9.9 \times 10^{-8}$ & $2.6 \times 10^{-7}$ & $6.0 \times 10^{-11}$ & $7.4 \times 10^{-11}$ & $1.7 \times 10^{-10}$ \\
$\mathrm{CO}$ & 1.15 & $1.3 \times 10^{-6}$ & $5.4 \times 10^{-7}$ & $2.7 \times 10^{-7}$ & $1.1 \times 10^{-10}$ & $1.1 \times 10^{-11}$ & $6.1 \times 10^{-10}$ \\
$\mathrm{ONe}$ & 1.15 & $8.0 \times 10^{-7}$ & $6.8 \times 10^{-7}$ & $7.9 \times 10^{-7}$ & $1.6 \times 10^{-11}$ & $6.4 \times 10^{-9}$ & $2.1 \times 10^{-8}$ \\
$\mathrm{ONe}$ & 1.25 & $6.0 \times 10^{-7}$ & $7.7 \times 10^{-7}$ & $6.9 \times 10^{-7}$ & $1.2 \times 10^{-11}$ & $5.9 \times 10^{-9}$ & $1.1 \times 10^{-8}$ \\
\hline
\end{tabular}
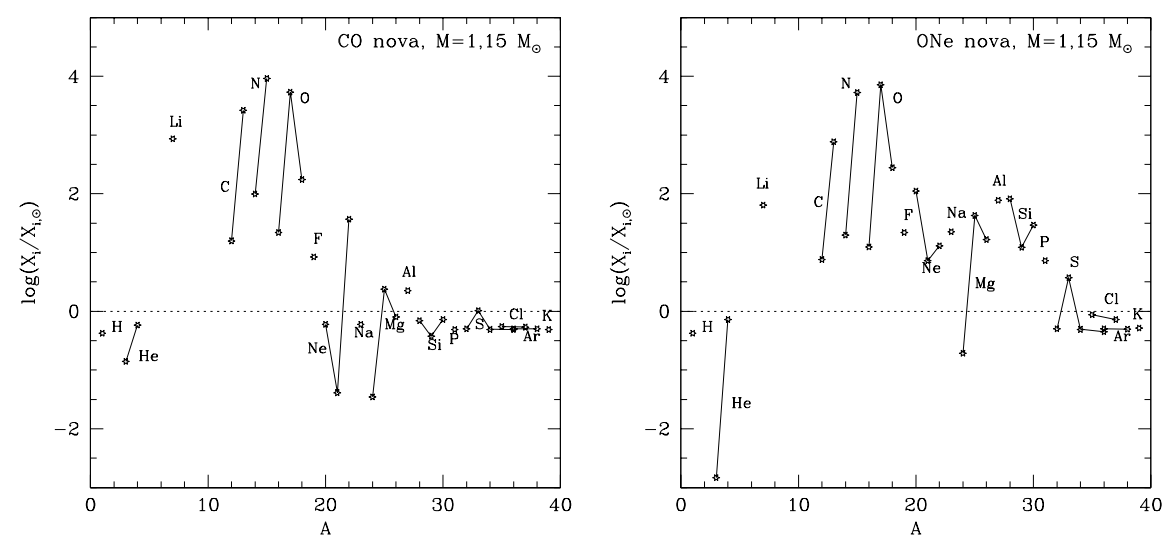

Figure 1. Overproduction factors relative to solar abundances, versus mass number for: (left) $\mathrm{CO}$ nova with $\mathrm{M}=1.15 \mathrm{M}_{\odot}$, (right) $\mathrm{ONe}$ nova with $\mathrm{M}=1.15 \mathrm{M}_{\odot}$

kpc with INTEGRAL, but it has very short duration and appears very early, even before optical detection. Detection of novae with INTEGRAL would provide a direct proof of the thermonuclear runaway model.

\section{Acknowledgments}

Research partially supported by the CICYT (ESP98-1348, PB98-1183-C03-02 and PB98-1183-C03-03) and by the AIHF1999-0140. 


\section{References}

1. S. Starrfield, J.W. Truran, W.M. Sparks and G.S. Kutter, ApJ 176, 169 (1972).

2. S. Starrfield, W.M. Sparks and J.W. Truran, ApJS 28, 247 (1974).

3. S. Starrfield, W.M. Sparks and J.W. Truran, ApJ 192, 647 (1974).

4. W.M. Sparks, S. Starrfield and J.W. Truran, ApJ 208, 819 (1976).

5. S. Starrfield, J.W. Truran and W.M. Sparks, ApJ 226, 186 (1978).

6. D. Prialnik, M.M. Shara and G. Shaviv, A\&A 61, 363 (1978).

7. J. Audouze, J.W. Truran and B.A. Zimmerman, ApJ 184, 493 (1973).

8. B. Lazareff, J. Audouze, S. Starrfield and J.W. Truran, ApJ 228, 875 (1979).

9. S. Amari, X. Gao, L. Nittler, E. Zinner, J. José, M. Hernanz and R. Lewis, $A p J$ in press, astro-ph/0012465 (2001).

10. J. José, M. Hernanz, S. Amari and E. Zinner, these proceedings.

11. R.D. Gehrz, J.W. Truran, R.E. Williams and S. Starrfield, Publ. Astr. Soc. Pac. 110, 3 (1998).

12. S. Starrfield, W.M. Sparks and J.W. Truran, ApJ 303, L5 (1986).

13. J.W. Truran and M. Livio, ApJ 308, 721 (1986).

14. M. Livio and J.W. Truran, ApJ 425, 797 (1994).

15. S.A. Glasner, E. Livne and J.W. Truran, ApJ 475, 754 (1997).

16. A. Kercek, W. Hillebrandt and J.W. Truran, $A \& \mathcal{E} A$ 337, 379 (1998).

17. A. Kercek, W. Hillebrandt and J.W. Truran, $A \& S A$ 345, 831 (1999).

18. D. Prialnik and A. Kovetz, ApJ 445, 789 (1995).

19. A. Kovetz and D. Prialnik, ApJ 477, 356 (1997).

20. J. José and M. Hernanz, ApJ 494, 680 (1998).

21. M. Politano, S. Starrfield, J.W. Truran, A. Weiss and W.M. Sparks, ApJ 448, 807 (1995).

22. S. Starrfield, J.W. Truran, M.C. Wiescher and W.M. Sparks, MNRAS 296, 502 (1998).

23. M. Hernanz, J. José, A. Coc and J. Isern, ApJ 465, L27 (1996).

24. S. Starrfield, J.W. Truran, W.M. Sparks and M. Arnould, ApJ 222, 600 (1978).

25. D. Romano, F. Matteucci, P. Molaro, P. Bonifacio, A $\& A$ 352, 117 (1999)

26. J. Gómez-Gomar, M. Hernanz, J. José and J. Isern, MNRAS 296, 913 (1998).

27. M. Hernanz, J. José, A. Coc, J. Gómez-Gomar and J. Isern, ApJ 526, L97 (1999).

28. J. José, A.Coc and M. Hernanz, ApJ 520, 347 (1999).

29. A. Coc, M. Hernanz, J. José and J.P. Thibaud, $A \& A$ 357, 561 (2000).

hernanz: submitted to World Scientific on November 19, 2018 